\title{
Towards an ethics of Al in Africa: rule of education
}

\author{
Sountongnoma Martial Anicet Kiemde ${ }^{1}\left[\right.$ ] Ahmed Dooguy Kora ${ }^{1}$
}

Received: 14 July 2021 / Accepted: 22 September 2021 / Published online: 4 October 2021

(c) The Author(s), under exclusive licence to Springer Nature Switzerland AG 2021

\begin{abstract}
First, this paper reviews the work on the ethics of artificial intelligence in Africa by researchers from Africa and the diaspora in a concept marked by a majority occidental contribution. Many African and diaspora researchers have proposed solutions for the development of ethical and responsible AI in Africa, but the proposed solutions are difficult to implement without the integration of ethical concepts in the training of actors. We show how education can be used to facilitate the development of responsible artificial intelligence in Africa. Through the introduction of ethics courses in academic training and capacity building of artificial intelligence development actors through research on responsible artificial intelligence in Africa, education will facilitate the integration of African ethical values and the development of responsible artificial intelligence through the diversification of artificial intelligence teams.
\end{abstract}

Keywords Africa $\cdot$ Artificial intelligence $\cdot$ Education $\cdot$ Ethics $\cdot$ Responsible AI

\section{Introduction}

In the face of massive awareness of the unintended negative consequences [1] of AI applications, the amount of work being done in AI ethics [2] continues to grow. This work is mainly led by AI research institutes, such as the Montreal Institute for AI [3], universities [4], companies, such as Microsoft [5] or BMW (ref), organizations, such as the European Commission's Independent High Level Expert Group on Artificial Intelligence which published its ethical guidelines for trustworthy AI in early 2019 [6] or the OECD principles on AI (ref OECD 2019), and government initiatives, such as the White House principles on AI [7]. Some recommendations have been issued by professional organizations, like the IEEE Standards Association [8] or ACM (Association of Computing Machinery).

Researchers were able to identify 84 documents containing ethical principles or guidelines for $\mathrm{AI}$ and found a significant increase in the number of publications by $88 \%$ from 2016 to 2019 [9] to be precise. Unfortunately, the contribution of Africa in the literature of AI ethics is very weak. While there are over $160 \mathrm{AI}$ ethics guidelines from around

Sountongnoma Martial Anicet Kiemde

kiemde.anicet97@gmail.com

1 Laboratoire e-Inov, Ecole Supérieure Multinationale des Télécommunications, Dakar, Senegal the world [10], the predominance of Western input on AI ethics guidelines can lead to a dominance of Western values and vision on AI ethics. Western values are still not universal. Moreover, the applications of AI are not only in the West but in the whole world. Africa is the continent that should benefit more from AI for its development despite an ecosystem that is not favorable to the development of AI on the continent [11], 12. Unfortunately, ethical issues are rarely on the agenda of discussions on AI in Africa. Thus, despite the numerous contributions to the debate on the ethics of AI at the global level, the contribution of African researchers and the diaspora remains very low. In this article we present a brief overview of the state of the art of AI on the continent. We also present the state of the art of work on the ethics of $\mathrm{AI}$ on the continent. Finally, the role of education, research, culture, and policies for responsible $\mathrm{AI}$ is presented.

\section{Contexte}

According to a PWC study, the added value of AI technologies in Africa will be \$1.2trn [13]. AI technologies could be used to solve several development problems on the continent [14]. Several African countries have begun to explore AI to meet their development needs. In the health field in South Africa, AI technologies are being used for rapid HIV testing [15]. In Uganda, AI is being used to advise individuals 
or emergency vehicles on optimal routes, dynamically redeploying a limited number of traffic police officers and analyzing possible reconfigurations of the road network to eliminate bottlenecks [16]. In Kenya, for example, the World Wildlife Fund for Nature (WWF) supports the use of an AI device with drones. After nine months, more than a dozen hunters had been apprehended in the Maasai Mara. WWF received a \$5 million grant from Google.org to use this AIpowered device to protect wildlife [17]. AI is used in Africa to help farmers improve crop yields. A more exhaustive list of different solutions based on AI technologies is presented in [18] and [12]. However, most of the initiatives and funding for the development of AI in Africa come from abroad. The continent receives several grants to facilitate the application and development of AI. IBM has committed $\$ 100$ million to its Lucy project to help improve infrastructure in Africa [19]. Much of Africa's digital infrastructure and ecosystem is controlled and managed by Western monopoly powers, such as Facebook, Google, Uber, and Netflix [20] or Chinese technology giants, like Huawei. Senegal inaugurated in June a datacenter to host all available data owned by the state with the help of the Chinese group Huawei [21]. In addition to initiatives, many deployed solutions with high demographic impact are coming from abroad, like China. For example, in March 2018 Chinese AI startup CloudWalk Technology, based in Guangzhou, signed an agreement with the government of Zimbabwe to deploy facial recognition technology and a surveillance system in Zimbabwe's cities and public transportation system there, including smart financial systems (to integrate finance with technology), and airport, railway, and bus station security. Similar agreements have been signed in Angola and Ethiopia [22].

However, AI can prove to be a tool for cyber colonization of the continent. For example in [23], Africa is seen by China as a test laboratory for improving its surveillance technologies. AI can be used by an authoritarian government for bad governance and targeting political opponents and reinforcing discrimination [24]. The lack of a regulatory or ethical framework may pose a data sovereignty problem for Zimbabweans.

According to the main AI readiness indices based on three key dimensions, namely, government, technology sector, and data and infrastructure, African countries score low [25]. The top five African countries in the Government AI 2020 global ranking are Mauritius (45th in the world), South Africa (59th), Seychelles (68th), Kenya (71st), and Rwanda (87th). However, on the AI readiness sub-index responsible for the government AI readiness index, Senegal ranks ninth. The report notes recent government progress in AI readiness in Tunisia and Egypt.

At the policy level, only 17 of the 55 African Union (AU) member states had enacted "comprehensive data protection and privacy legislation," according to a Global Information
Society Watch 2019 study [26]. These are Angola, Benin, Burkina Faso, Cape Verde, Côte D 'Ivoire, Gabon, Ghana, Lesotho, Madagascar, Mali, Mauritius, Morocco, Senegal, Seychelles, South Africa, Tunisia, and Western Sahara. The first AI strategy in Africa is that of Mauritius. The country has also announced the establishment of a National AI Council [25].

\section{State of the art}

Faced with an awareness of the possible dangers of the application of AI in Africa in the absence of a regulatory framework, researchers in Africa and the diaspora have made contributions to raise awareness and to put in place regulations for responsible AI.

In [27], the author proposes six principles for AI ethics in Africa. These include introducing safeguards to balance the opportunities and risks of AI, defining African AI values and aligning AI frameworks with these values, practicing equitable and socially responsible AI, building inclusive partnerships based on community and co-creation, and adopting an adaptive, open and humble approach. This report calls for a code of ethics that integrates several disciplines, such as social sciences and legal sciences. What emerges is a political commitment and the development of a strong value proposition at the public policy level in order to generate public interest in AI. In order to avoid or reduce the potential risks of $\mathrm{AI}$ in Africa, $\mathrm{AI}$ policy formulations must consider ethical and socio-cultural aspects.

In [28], the authors propose that for AI to work in Africa, local researchers should be involved in the development of AI based on African data sets. It recommends an awareness of the development process and the limitations of imported AI technologies. There was also discussion of making African data available to local researchers and companies working with important technologies. All this in an environment of data privacy. Policymakers should promote regulations that promote ethics by design through additional checks and balances in systems that use AI. He suggests three key recommendations, namely, that not all $\mathrm{AI}$ is the same, policies must be well informed and aligned with the needs and values of the cultures represented in each African nation, as well as provide a holistic vision for a better future, and AI allows for solutions to be created in a new way that can mask the underlying logic. He recommends avoiding using a strategy of regulations based on vague ethical ideas as this would not only be ineffective but could also undermine the innovation process.

In [29], the author appeals not to create psychosis among our policymakers or developers of AI solutions that will cause them to under-exploit the benefits of AI for Africa's sustainable development but rather to warn of the possible 
risks of using $\mathrm{AI}$ without proper regulation. This regulation should not be too onerous. The risk is great that AI will be underutilized for fear of overuse or abuse.

In [30], after exploring the role and means by which Africa can best exploit the opportunities offered by intelligent automation and robotics, the author highlights strategies to offset threats posed by global factors, such as premature deindustrialization.

In [31], the authors critically examine a proposal to ensure that decision-making systems are fair, equitable, and intelligible - that they adopt an explicability principle to generate specific recommendations - to assess whether the principle should be adopted in an African research context. The authors argue that an explainability principle can not only contribute to the responsible and thoughtful development of AI that is sensitive to African interests and values but can also advance some of the computational challenges of machine learning research.

In [32], the author illustrates how the AI invasion of Africa echoes the exploitation of the colonial era. The author concludes by describing a vision of AI rooted in the needs and interests of the local community. She questions the indiscriminate importation of the latest state-of-the-art machine learning systems or some other Western AI tools without questioning the purpose and contextual relevance, who benefits and who might be disadvantaged by the application of such tools.

In [33], the authors provide an overview of key elements of AI deployment in Africa, key benefits and challenges of $\mathrm{AI}$ in African contexts, and key policy dimensions of AI for the continent. According to the authors, If African countries want to build inclusive AI ecosystems, informed policymaking is essential.

In [34], the author argues for a broader perspective on the ethics of AI, including the contribution of Africa. He demonstrates that socio-cultural characteristics influence our conceptions of ethics and, in this case, AI ethics. It proposes a roadmap on how we can better engage in AI ethics and the development of guidelines that have far-reaching effects.

In [35], based on the four areas of innovation, capacity building, policy, and infrastructure provide an overview of the state of AI in African contexts and propose a general roadmap of key activities needed for Africa to position itself to better exploit responsible AI technologies.

In [36], the author reviews the interference of $\mathrm{AI}$ in the enjoyment of human rights and fundamental freedoms. He then explains why it is important to move towards regulation in this field in Morocco. The author proposes general principles for regulating $\mathrm{AI}$ in order to make it ethical, responsible and respectful of human rights.

The enforcement phase of ethics has been discussed in [29]. The authors believe that regulation of AI should begin before it matures. However in [27], ethics must come before, during, and after the law and support the policy and implementation of AI. In [36], the author proposes the co-regulation of AI for Morocco. Stakeholders (including technology companies, universities, and associations), as well as the government, are called upon to launch a broad consultation on an ethical code for AI.

Many African and diaspora researchers have proposed solutions for the development of ethical and responsible AI in Africa, but the proposed solutions are difficult to implement without the integration of ethical concepts in the training of actors.

\section{Education and research for Al ethics}

Education has a prominent role to play in developing AI ethics on the continent through the democratization of technology. Educating future members of the AI community, as well as other stakeholders about the impact and danger of $\mathrm{AI}$, could occur in part through the more comprehensive and systematic inclusion of AI ethics in educational programs [37]. However, in a survey of 2360 students, academics, and data science professionals by the software company Anaconda, only $18 \%$ of data science students are familiar with $\mathrm{AI}$ ethics and $15 \%$ of instructors and professors reported that they teach AI ethics [38]. According to a UNESCO survey on AI in Africa, 26 countries report a skills gap in dealing with the ethical implications of AI, while only 6 countries reported having the necessary capacity on this issue [39].

\section{A. Integration of ethical courses in the training offers}

From the development of training curricula in schools and universities to capacity building programs for staff in institutions, training on the ethical issues and risks associated with AI must be integrated. In these trainings, it is important to highlight that AI applications can go against our moral principles, change our way of life, especially our relationships with each other and the way we work or collaborate. For example, in capacity building for justice personnel, it is necessary to prepare the African justice system to deal with legal issues when mistakes are made, or harm is caused by AI technologies on the population. This must be done by training the different entities of the justice system on the legal issues of $\mathrm{AI}$, the establishment or integration in the penal code of the sanctions to be afflicted, and compensation and responsibilities in case of harm. The results of the survey show that $78 \%$ of the participating countries $(25$ countries) have requested knowledge exchange activities and $90 \%$ (29 countries) have requested assistance in training their judicial officials [39].

These statistics demonstrate the real need for training and capacity building of justice actors. Also, there is a 
need to equip the judicial system with high technical skills and resources to facilitate the analysis of algorithmic decisions. These resources and skills will be useful for the right to explanation and audit, very important for judges and lawyers.

For the training of AI solution developers, beyond mathematical and programming skills, it is important to integrate existing mathematical and technical concepts that surround ethical issues, such as algorithmic fairness, transparency, or explicability of models. For example, when teaching mathematical derivations of a linear regression function for supervised learning in AI, we can also mention the use of disparate impact as a metric to evaluate the fairness of the output in the hope that we are getting closer to a "correct" and "fairer" result [37].

For schools and universities, the training of engineers, AI developers should lead them to cultivate technosocial virtue. The techno-social approach must consider the beneficiaries of AI technology with a study of the impact of the technology in their lives. Also, access to training will facilitate a diversification of AI teams. Diversity must be at the core of the IA ethos. IA development teams should be made up of many people from different cultural, ethnic, gender, and religious backgrounds. This may seem difficult to achieve because there are thousands of different ethnicities and religions on the continent, but it is still important to diversify the teams as much as possible. The study, which was conducted by The AI Now Institute at New York University, found that the AI field, which is predominantly white and male, risks reproducing or perpetuating historical biases, and power imbalances [40].

Training is not only limited to universities, schools, and institutions but also to the population, the users of AI solutions. The training of the population, especially those under 50, on the fundamentals of AI, such as the training organized in several countries on the continent on financial inclusion.

B. Integration of African ethical values through education

An approach based on African values and realities will provide a foundation for ethical AI in Africa and facilitate the deployment and development of AI on the continent. There are significant differences between what is ethical in Africa and what is ethical in the West. Artificial intelligence is the work of human intelligence. Westerners tend to see their view as the right one and the way everyone should live, which is of course ignorant and wrong. The current development of artificial intelligence depends on the traditional Western vision of the person as rationality [41]. It should not logically have the free will to make decisions without human validation or without being in accordance with inter-human moral principles. Therefore, AI should not dominate humans, but rather humans should control the decisions made by AI with the possibility of rectifying the decision.

The values or principles of AI ethics must be consistent with the moral values or ethics of Africa. AI ethics must be human ethics [42]. In this sense, the training of actors will facilitate the contribution and adaptation of African moral values to the ethics of AI in Africa. In addition, inclusive training will facilitate the development of skills from all socio-cultural backgrounds which will facilitate the diversity of the AI teams. Remember that Africa is a continent of many cultures, many ethnicities, and many religions. In the 54 African nations, 3000 different indigenous groups live, including Berbers and Zulus, Yorubas and Igbos, Fulanis and Kikuyus, and, of course, many others [43]. In addition, there are differences in skin color. In sub-Saharan Africa, most of the population is black. Several AI algorithms have been found to discriminate against blacks peoples [44].

Ubuntu lessons in Africa, ranging from "Seeing yourself in others" to "Learning to listen allows you to hear better" Ubuntu - I am because you are. The Ubuntu of sub-Saharan African origin combines human principles with brotherhood. The development of AI must be in accordance with African values about man. Those that put human relationships at the center and not individualism. Unfortunately, a rational view of people has always been marked by contradictions, exclusions, and inequalities. The development of AI based on data generated by such a vision will contribute to increase the already existing inequalities.

AI must not promote the trivialization of evil or even trivialize evil, which is an increasingly common phenomenon in Africa. It is important that the ethical framework of AI does not rely solely on a Western vision that may ignore the cultural values of marginalized communities, especially black people (who are of African descent). African values can be incorporated into the creation and use of AI technologies. These values emphasize rationality and love for others. These values do not allow just for the individual but rather for the ability of people to coexist with others.

\section{The research}

The contribution of researchers on the continent is weak on the issues of equity, transparency, and auditing of AI algorithms. A strong involvement of African researchers could be of great help for ethical AI in Africa. Research should also focus more on the interactions between humans and AI. For AI to work in Africa, local researchers need to be involved in the development of new technologies, with African data sets informing this development. Initiatives to encourage research in responsible AI are being deployed by AI4D. The AI Development Initiative has launched a call for propos- 
als entitled "Towards Responsible AI, for African AI" as part of the AI for Development program funded by the IDRC and SIDA (Swedish International Development Cooperation Agency). We must therefore stimulate research on the ethics of $\mathrm{AI}$ and this through consistent funding of AI research in Africa. AI4D Africa is a 4-year, C\$20 million partnership to support the creation of an ecosystem that fosters the responsible and local development and deployment of AI through investments in three key areas: innovation, policy research, and skills [45]. However, AI research on the continent must be funded mostly by state public funds to avoid or reduce the influence of large technology companies or private investors. Education is a key to ensuring that the principles of AI ethics are implemented.

\section{Conclusion}

The impact of AI in society is an absolute certainty that no one can doubt, whether it is negative or positive. It is up to us to maximize the positive impact of AI while minimizing its negative impact. Focusing on the negative impact of AI would lead us to underutilize the potential that AI offers. Focusing only on the positive impacts would lead us to ignore or neglect the negative consequences and delay the implementation of a regulatory framework for $\mathrm{AI}$ in our societies.

An ethical framework for AI will enable the continent to better leverage the many benefits that AI offers for the development of the continent. Such a framework would anticipate the potential dangers of $\mathrm{AI}$ and help create possible preventive solutions and the establishment of a sound policy for the development of sustainable AI for the continent.

AI is a technological innovation. Not every technological innovation is a social improvement. Africa needs social improvement more than technological innovation. Only with sufficient focus on AI ethics we can make the technological innovation of AI into a social improvement for Africa.

The fundamental limitation of this study is that it does not provide a much more specialized analysis of one country and may lead to a risk of generalization across such a vast continent, characterized by very different social, cultural, and political arrangements within its constituent countries.

In our future work we will focus on Francophone African countries, where the contribution and debate on the ethics of $\mathrm{AI}$ and responsible AI remain mixed.

\section{References}

1. Veale, M., Binns, R.: Fairer machine learning in the real world: Mitigating discrimination without collecting sensitive data. Big Data Soc 4, 2 (2017). https://doi.org/10.1177/2053951717743530

2. Paula, B.: Towards a code of ethics for artificial intelligence, $1 \mathrm{st}$ edn. Springer International Publishing (2017)
3. Université de Montréal, "La Déclaration de Montréal en IA responsable." [Online]. https://www.declarationmontreal-iares ponsable.com/la-declaration

4. Montreal Declaration, "The declaration," (2017)

5. Microsoft: Microsoft AI principles. (2017), [Online]. https://www. microsoft.com/en-us/ai/our-approach-to-ai

6. High-Level Expert Group on AI, "Ethics Guidelines for Trustworthy AI," (2019)

7. Vought, R. T.: Guidance for regulation of artificial intelligence applications introduction. 3967(13), 1-11 (2009)

8. IEEE Standards Association, "The IEEE global initiative on ethics of autonomous and intelligent systems," (2019). https://standards. ieee.org/industry-connections/ec/autonomous-systems.html

9. Jobin, A., Ienca, M., Vayena, E.: The global landscape of AI ethics guidelines. Nat. Mach. Intell. 1(9), 389-399 (2019) https://doi.org/ 10.1038/s42256-019-0088-2

10. Leonard H.V.T., Sebastian, G.: In the realm of paper tigers exploring the failings of AI ethics guidelines. (2020). https://algor ithmwatch.org/en/ai-ethics-guidelines-inventory-upgrade-2020/

11. Arakpogun, E., Elsahn, Z., Olan, F., Elsahn, F.: Artificial intelligence in africa: challenges and opportunities. pp. 375-388 (2020)

12. Anicet K.S.M., Dooguy K.A.: The challenges facing the development of AI in Africa. (2020) https://doi.org/10.1109/ICATM RI51801.2020.9398454

13. Verweij, G., Rao, A.: Sizing the prize: what's the real value of AI for your business and how can you capitalise?," PwC. 32, (2017)

14. Andersen, L.: Artificial intelligence in international development: avoiding ethical pitfalls. J. Public Int. Aff. (2019) [Online] https:// jpia.princeton.edu/news/artificial-intelligence-international-devel opment-avoiding-ethical-pitfalls

15. Turbé, V., et al.: Deep learning of HIV field-based rapid tests. Nat. Med. (2021) https://doi.org/10.1038/s41591-021-01384-9

16. Gul, E.: Is artificial intelligence the frontier solution to global south's wicked development challenges? Towards Data Sci https:// towardsdatascience.com/is-artificial-intelligence-the-frontier-solut ion-to-global-souths-wicked-development-challenges-42062 $21 \mathrm{a} 3 \mathrm{c} 78$

17. Wildlife Crime Technology. "Wildlife Crime Technology Project I Projects | WWF". https://www.worldwildlife.org/projects/wildl ife-crime-technology-project

18. Brandusescu, A., Freuler, J.O., Thakur, D.: Artificial intelligence Starting the policy dialogue in Africa. Artif. Intell. pp. 1-8, (2017). [Online] www.webfoundation.org.

19. IBM.: "IBM research: IBM brings Watson to Africa for Project Lucy. IBM (2014) http://www.research.ibm.com/labs/africa/proje ct-lucy.shtml.

20. Micheal, K.: "Digital colonialism is threatening the Global South," Aljazeera. p. Al Jazeera, (2019). [Online] https://www. aljazeera.com/opinions/2019/3/13/digital-colonialism-is-threa tening-the-global-south/

21. Nirina, "Au Sénégal, un Data Center d'Etat accueilli comme une révolution," Lebigdata

22. Gwagwa, A., Garbe, L.: "Exporting repression? China's artificial intelligence push into Africa," Council on foreign relations. p. Counc. Foreign relations, [Online] https://www.cfr.org/blog/expor ting-repression-chinas-artificial-intelligence-push-africa

23. Gravett, W.H.: Digital coloniser? China and artificial intelligence in Africa. Survival (Lond) 62(6), 153-178 (2020) https://doi.org/ 10.1080/00396338.2020.1851098

24. Berisha, V.: "AI as a threat to democracy : towards an empirically grounded theory AI as a threat to democracy : towards an empirically grounded theory. Visar berisha autumn 2017 Supervised by Professor Joakim Palme,” 2017, 0-64 (2019) https://doi.org/10. 13140/RG.2.2.33108.24960.

25. I. Development.: AI Readiness Index 2020 full report. (2020) 
26. G. I. S. Watch: Where are we watching. Global Information Society Watch (2019)

27. Arthur, G.: Reccomendations on the inclusion sub- Saharan Africa in Global AI Ethics. pp. 1-13, (2019)

28. Sallstrom, L., Morris, O., Mehta, H.: Artificial Intelligence in Africa's Healthcare: Ethical Considerations. ORF Issue Brief No. 312. Observer Research Foundation (2019)

29. Floridi, L., et al.: AI4People — an ethical framework for a good AI society: opportunities, risks, principles, and recommendations. Minds Mach. 28(4), 689-707 (2018). https://doi.org/10.1007/ s11023-018-9482-5

30. Vernon, D.: Robotics and artificial intelligence in Africa [Regional]. IEEE Robot. Autom. Mag. 26(4), 131-135 (2019) https://doi.org/10.1109/MRA.2019.2946107

31. Carman, M., Rosman, B.: Applying a principle of explicability to AI research in Africa: should we do it? Technol. Ethics Inf. (2020) https://doi.org/10.1007/s10676-020-09534-2

32. Birhane, A.: Algorithmic colonization of Africa. SCRIPT-ed 17(2), 389-409 (2020) https://doi.org/10.2966/scrip.170220.389

33. Gwagwa, A., Kraemer-Mbula, E., Rizk, N., Rutenberg, I., De Beer, J.: Artificial intelligence (AI) deployments in Africa: benefits, challenges and policy dimensions. African J. Inf. Commun. 26, 1-28 (2020) https://doi.org/10.23962/10539/30361

34. Segun, S.T.: Critically engaging the ethics of AI for a global audience. Ethics Inf. Technol. (2020) https://doi.org/10.1007/ s10676-020-09570-y

35. Gwagwa, A., Kachidza, P., Siminyu, K., Smith, M.: Responsible artificial intelligence in sub-saharan Africa: landscape and general state of play. AI4D Africa (2021)

36. Bensalah, M.: Toward an ethical code of $\mathrm{AI}$ and human rights in Morocco. (2021) https://doi.org/10.13140/RG.2.2.26500.45446/1
37. Borenstein, J., Howard, A.: Emerging challenges in AI and the need for AI ethics education. AI Ethics 1(1), 61-65 (2021). https:// doi.org/10.1007/s43681-020-00002-7

38. Macaulay, T.: Study: only $18 \%$ of data science students are learning about AI ethics. The Next Web. (2020) [Online] https://thene xtweb.com/neural/2020/07/03/study-only-18-of-data-scientistsare-learning-about-ai-ethics/

39. UNESCO.: Artificial intelligence needs assessment survey in Africa. (2021)

40. Myers-West, S., Whittaker, M., Crawford, K.: Discriminating systems: gender, race and power in AI. (2019)

41. Sabelo, M.: "Carr center for human rights policy from rationality to relationality : ubuntu as an ethical and human intelligence governance. Carr Cent. Hum. Rights Policy. 2020-009 (2020)

42. Boddington, P.: AI and moral thinking: how can we live well with machines to enhance our moral agency? AI Ethics 1(2), 109-111 (2021) https://doi.org/10.1007/s43681-020-00017-0

43. Yembra, N., et al.: Tech in Africa. The Generalist

44. Kiemde, S.M.A., Kora, A.D.: Fairness of machine learning algorithms for the black community. IEEE Internat Symp Technol Soc (ISTAS) 2020, 373-377 (2020) https://doi.org/10.1109/ISTAS 50296.2020.9462194

45. AI4D: AI4D Afrique

Publisher's Note Springer Nature remains neutral with regard to jurisdictional claims in published maps and institutional affiliations. 